\title{
Observation of two visible Dicke-superradiant transitions in atomic europium (*)
}

\author{
C. Brechignac and $\mathrm{Ph}$. Cahuzac \\ Laboratoire Aimé Cotton, C.N.R.S. II, Bâtiment 505, 91405 Orsay, France
}

(Reçu le $1^{\mathrm{er}}$ décembre 1978, révisé le 22 janvier 1979, accepté le 29 janvier 1979)

\begin{abstract}
Résumé. - Nous présentons ici la première mise en évidence expérimentale de deux émissions superradiantes dans le domaine visible pour les raies de l'europium à $\lambda=557,7 \mathrm{~nm}$ et $\lambda=545,3 \mathrm{~nm}$. Le caractère superradiant de ces transitions est démontré en étudiant la variation de l'intensité de l'impulsion, de sa largeur et de son retard en fonction de la densité atomique. Le mécanisme particulier de l'inversion de population est dû à l'existence d'une émission superradiante infrarouge à $\lambda=1759,6 \mathrm{~nm}$.
\end{abstract}

Abstract. - We report the first experimental evidence of visible superradiance for the europium lines at $\lambda=557.7 \mathrm{~nm}$ and $\lambda=545.3 \mathrm{~nm}$. The superradiant character of these lines is checked by studying the variation of pulse heights, widths and delays versus atomic density. We show that the peculiar population inversion mechanism is essentially due to the existence of a strong superradiant infrared emission at $\lambda=1759.6 \mathrm{~nm}$.

During the last few years observation of superradiant or superfluorescence light pulses in the infrared or near infrared range has been reported by several groups [1-7]. Critical conditions have to be satisfied for the observation of such an effect. Especially the characteristic coupling time

$$
\tau_{\mathbf{R}}=8 \pi / \gamma_{\mathrm{ab}} n L \lambda^{2}
$$

must be shorter than the dephasing time $T_{2}^{*}$ of atomic dipoles [7-9]. In this formula $\gamma_{a b}$ is the transition probability and $\lambda$ the wavelength of the observed line, $n$ is the initial population inversion density and $L$ the length of the superradiant volume. Consequently achievement of superradiance is subject to a threshold condition upon $n$. As is well known the superradiance emission starts with a delay $\tau_{D}$ proportional to $\tau_{R}$ after the excitation, and generally $\tau_{D}$ is one or two orders of magnitude larger than $\tau_{R}$. In the far infrared range superradiance takes place with a delay $\tau_{D}$ on a microsecond time-scale and there are no serious experimental difficulties presenting this effect being observed. On the contrary, in the visible range superradiance takes place with delay $\tau_{\mathrm{D}}$ of a few nanoseconds and the excitation process leading to a total population inversion has to be

(*) La version française de cet article est publiée dans le ${ }^{\circ} 4$ des Comptes Rendus de l'Académie des Sciences, Série B, du 29 janvier 1979. achieved in a comparable time scale. This can be obtained by using short laser pulses now available. We report here the first experimental evidence of visible superradiance in europium vapour.

The relevant levels of the studied system are shown in figure 1 . The system is prepared in the $5 \mathrm{~d} 6 \mathrm{p}^{10} \mathrm{D}_{9 / 2}$ state as follows :

(i) the $6 \mathrm{~s} 6 \mathrm{p}^{8} \mathrm{P}_{9 / 2}$ level is resonantly excited by using a pulsed dye laser at the blue wavelength $\lambda_{1}=459.4 \mathrm{~nm}$;

(ii) radiative decay occurs at $\lambda=1759.6 \mathrm{~nm}$ towards the $6 \mathrm{~s} 5 \mathrm{~d}^{8} \mathrm{D}_{11 / 2}$ level;

(iii) resonant excitation of the $5 d 6 p^{10} D_{9 / 2}$ level

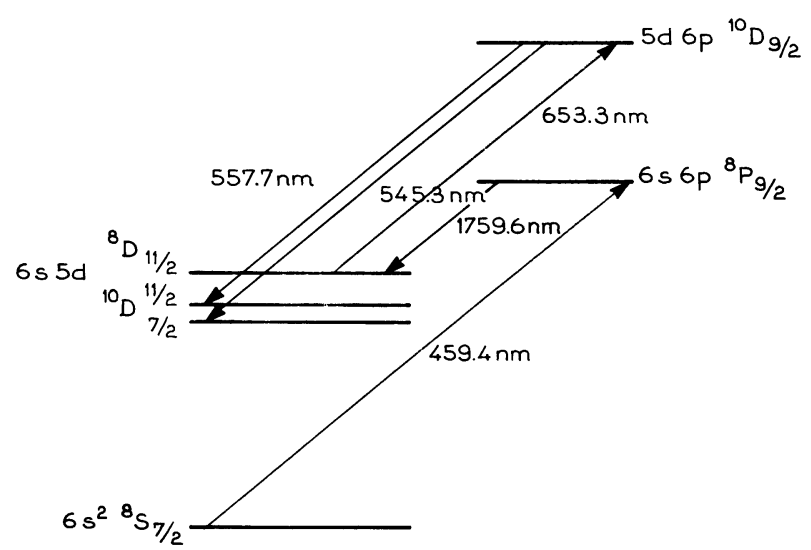

Fig. 1. - Simplified level scheme of superradiant system. 
is achieved by using another pulsed dye laser at the red wavelength $\lambda_{2}=653.3 \mathrm{~nm}$.

In fact the $1759.6 \mathrm{~nm}$ emission which populates the $6 \mathrm{~s} 5 \mathrm{~d}^{8} \mathrm{D}_{11 / 2}$ level is a superradiant (S.R.) emission ; it provides a fast and efficient excitation of this level $\left({ }^{1}\right)$. Although the superradiant nature of this infrared line seems not to be necessary in this process, it allows the depopulation of the resonant level towards the $6 \mathrm{~s} 5 \mathrm{~d}^{8} \mathrm{D}_{11 / 2}$ level faster than any other relaxation process. Since the pumping-rate by the red laser is also very efficient no significant population appears on the metastable $6 \mathrm{~s} 5 \mathrm{~d}^{10} \mathrm{D}_{J}$ levels. Large population inversion is built between $5 \mathrm{~d} 6 \mathrm{p}^{10} \mathrm{D}_{9 / 2}$ and metastable $6 \mathrm{~s} 5 \mathrm{~d}^{10} \mathrm{D}_{J}$ levels. Observation of visible S.R. lines at $\lambda=557.7 \mathrm{~nm}$ and $\lambda=545.3 \mathrm{~nm}$ for which there is no initial macroscopic polarization is possible because there is no competition with infrared transitions starting from the upper level.

The experimental set up includes two pulsed dye lasers pumped by the same nitrogen laser. They provide pulses with 5-6 ns duration and a few kilowatt peak power. Their bandwidth is typically $6 \mathrm{GHz}$. The europium vapour is provided by a thermo regulated heat-pipe-type cell. This cell is filled with argon as buffer gas in a pressure range 5-15 torr, much higher than the metallic vapour pressure. Both laser beams are overlapping and copropagating. They are focused in the oven and the laser flux density is of the order of $5 \mathrm{MW} / \mathrm{cm}^{2}$. The active region is a cylinder with $2 \mathrm{~cm}$ length and $0.04 \mathrm{~cm}$ diameter. The backward fluorescence is observed. A monochromator is used to discriminate and identify the studied fluorescence lines. Time-resolved studies are performed using a fast gate-detection including a boxcar integrator with a 350 ps sampling head following a fast detector (photomultiplier or Ge-Au).

With this set up we have analysed the nature of the observed lines. For the appropriate laser wavelengths a bright and directively emitted fluorescence is observed in forward and backward directions when the temperature of the cell provides a metallic vapour pressure larger than a few tenths of a torr. A spectral analysis shows the existence of the three intense lines at $\lambda=557.7 \mathrm{~nm}, \lambda=545.3 \mathrm{~nm}$ and $\lambda=1759.6 \mathrm{~nm}$, easy to identify as shown as figure 1 [11]. One can notice that the two visible lines sharing the same upper level have comparable transition probabilities. The infrared emission occurs even when the red laser is switched-off but no visible emission exists if $\lambda_{1}$ and/or $\lambda_{2}$ are off-resonance with the corresponding europium transitions.

We have studied the emitted pulses as a function of time. These pulses occur significantly above a threshold $n_{\text {th }}$ of the atomic density $n_{0}$ in the ground state.

$\left.{ }^{1}\right)$ This infrared transition has been already observed as a strong pulsed laser line [10].
We have investigated the variation of the pulse height $I_{\mathrm{S}}$ and of its delay and width $\tau_{\mathrm{D}}$ and $\tau_{\mathrm{w}}$ respectively versus $n_{0}$. These measurements have been performed essentially for the $557.7 \mathrm{~nm}$ line by varying the temperature of the cell. In a series of measurements the laser flux is kept constant therefore in the following we use equivalently $n$ or $n_{0}$. Qualitatively figure 2

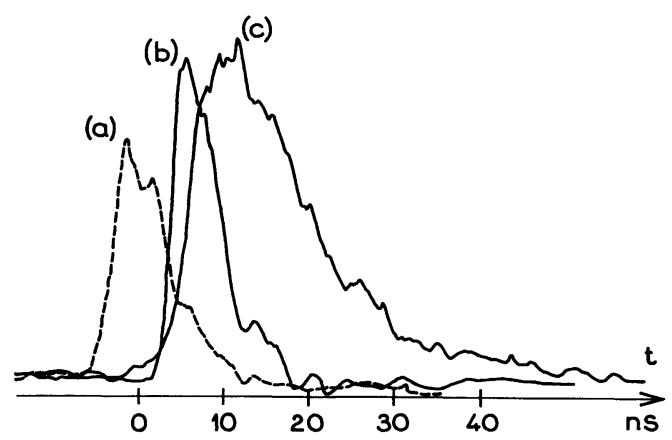

Fig. 2. - Time-evolution of the S.R. pulse at $\lambda=557.7 \mathrm{~nm}$ for two temperatures (trace $\mathrm{b}: 830^{\circ} \mathrm{C}$; trace $\mathrm{c}: 800^{\circ} \mathrm{C}$ ) compared with the time-evolution of the red laser pulse (trace a). The amplitude scales are arbitrary.

shows the time evolution of the red laser pulse (trace a) and the emitted pulse for two different temperatures (traces $b$ and $c$ ). This pulse occurs with a noticeable delay compared to the laser pulse. This delay increases when the temperature decreases and simultaneously the width of the pulse increases. Quantitatively the dependences on density are shown in figure 3 . We measured the delay times $\tau_{\mathrm{D}}$ in a density range $n_{0}=0.3$ to $1 \times 10^{16} \mathrm{~atm} / \mathrm{cm}^{3}, \tau_{\mathrm{D}}$ increasing linearly versus $n_{0}^{-1}$ from 5 ns to 13 ns (Fig. 3). For these measurements a part of the red laser pulse is used for synchronization of our gate detection. The error bar for the time-origin corresponds to the jitter of the trigger.

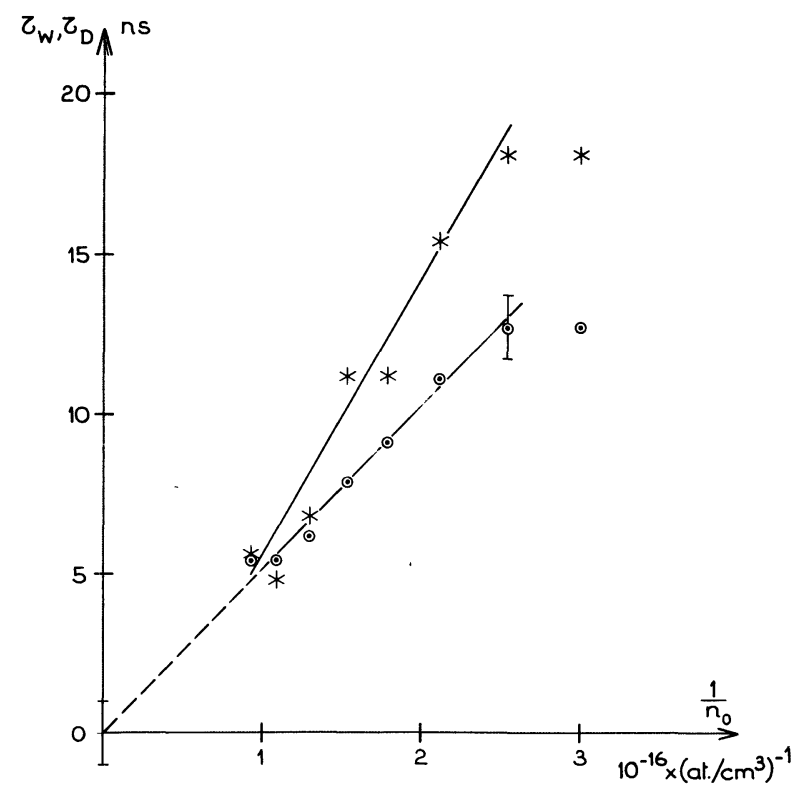

Fig. 3. - Dependence of $\tau_{\mathrm{D}}(\odot$ points $)$ and $\tau_{\mathrm{w}}(*$ points $) v s$. $n_{0}^{-1}$ for the line at $557.7 \mathrm{~nm}$. 
For the highest value of $n_{0}$, the delay $\tau_{\mathrm{D}}$ becomes so short that the emitted pulse occurs before the laser pulse stops. This situation corresponds to the lower limit for the observed delay. On the other hand for the lowest value of $n_{0}$ the delay is determined by the threshold condition.

In figure 3 is also plotted the variation of the pulse width $\tau_{\mathrm{w}}$ versus $n_{0}^{-1}$. A linear variation is observed. We have to notice that our time analysis includes integration over several pulses and so we integrate the statistical fluctuations; this leads to an experimental broadening.

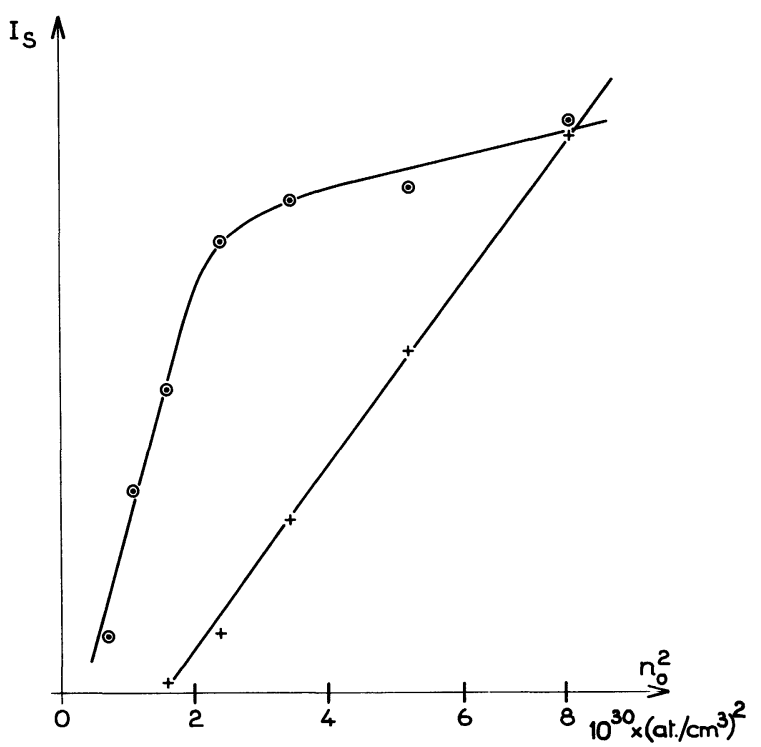

Fig. 4. - Dependence of the height of the S.R. pulses at $557.7 \mathrm{~nm}$ $(+)$ and $1759.9 \mathrm{~nm}(\odot) v s . n_{0}^{2}$.

In figure 4 are shown the pulse height $I_{\mathrm{S}}^{\mathrm{V}}$ for the $557.5 \mathrm{~nm}$ and $I_{\mathrm{S}}^{\mathrm{IR}}$.for $1759.6 \mathrm{~nm}$ lines against $n_{0}^{2}$. These measurements have been performed with simultaneous laser pulses. For the visible line $I_{\mathbf{S}}^{\mathbf{V}}$ increases linearly versus $n_{0}^{2}$ in the investigated density range and the threshold is observed. For the infrared S.R. pulse under the same experimental conditions the threshold is observed for a smaller value $n_{\text {th }}$ of $n$. Just above this threshold $I_{\mathrm{S}}^{\mathrm{IR}}$ increases linearly versus $n_{0}^{2}$ and for $n \gtrsim 2 n_{\mathrm{th}}$ the pulse height is saturated and does not behave like $n_{0}^{2}$. We have verified that under these conditions there is an overlap between the infrared S.R. pulse and the blue laser pulse. In these measurements we used two simultaneous dye laser pulses ; then an efficient population of the upper level $5 \mathrm{~d} 6 \mathrm{p}^{10} \mathrm{D}_{9 / 2}$ is achieved only when the infrared pulse saturates. Therefore, as is observed, the visible line appears. When the infrared emission is not saturated it is also possible to observe visible emission by using an optical delay for the red laser pulse against the blue one. We have experimentally checked that this delay must be larger than the delay of the infrared line. These observed variations for $I_{\mathrm{S}}, \tau_{\mathrm{D}}$ and $\tau_{\mathrm{w}}$ versus $n_{0}$ are in agreement with the expected variations for superradiant emissions in particular $I_{\mathrm{S}} \propto n^{2}$ characterizes collective emission. Assuming an efficiency of the pumping rate for the $5 \mathrm{~d} 6 \mathrm{p}^{10} \mathrm{D}_{9 / 2}$ level in the range $10^{-2}$ to $10^{-3}$ and using the longitudinal relaxation time $T_{1}=50 \mathrm{~ns}$ [12] one obtains a characteristic coupling time $\tau_{\mathrm{R}}$ in the range 0.02 to $0.2 \mathrm{~ns}$. One can notice that in our case the delay $\tau_{\mathrm{D}}$ is smaller than $T_{1}$ but larger than $T_{2}^{*}=0.5 \mathrm{~ns}$. Actually for an extended high gain medium the condition to be fulfilled for S.R. emission is $\tau_{\mathrm{D}}<\alpha l T_{2}^{*}$ where $\alpha l$ is the gain of the medium [13]. In our case the relationship $\alpha l=T_{2}^{*} / \tau_{\mathrm{R}}[1]$, leads to $\alpha l \gg 1$ as can be expected for such high gain medium.

To conclude, the experimental results presented here demonstrate the superradiant nature of the observed lines. In spite of the small value of $T_{2}^{*}$ in the visible range a total population inversion can be achieved. This population inversion is easily obtained via a large population density of the $6 \mathrm{~s} 5 \mathrm{~d}^{8} \mathrm{D}_{11 / 2}$ level. A simple mechanism to strongly populate this level is the use the S.R. infrared line. More generally such a process can be used to provide a strong population in metastable levels and this pure optical procedure has the advantage of being fast and selective. In other words as soon as we dispose of an efficient and fast pumping process, superradiance takes place before any other relaxation process and so it can be observed. During the course of this work many other europium lines in the visible range have been observed showing similar characteristics but involving a slightly different excitation process. A complete survey of these results and of the involved mechanism will be published in the near future.

\section{References}

[1] Skribanowitz, N., Herman, I. P., McGillivray, J. C. and Feld, M. S., Phys. Rev. Lett. 30 (1973) 309.

[2] Gross, M., Fabre, C., Pillet, P. and Haroche, S., Phys. Rev. Lett. 36 (1976) 1035.

[3] Flusberg, A., Mossberg, T. and Hartmann, S. R., Phys. Lett. 58A (1976) 373.

[4] Gibbs, H. M., VreheN, Q. M. F. and Hikspoors, H. M. J., Phys. Rev. Lett. 39 (1977) 547.

[5] Crubellier, A., Liberman, S. and Pillet, P., Phys. Rev. Lett. 41 (1978) 1237.

[6] Gounand, F., Hugon, M., Fournier, P. R. and Berlande, J., to be published in J. Phys. B.
[7] Pillet, P., Thèse de 3e cycle, Université de Paris VI, 1977, unpublished.

[8] Bonifacio, R. and Lugiato, L. A., Phys. Rev. A 11 (1975) 1507 and references therein.

[9] Dicke, R. H., Phys. Rev. 93 (1954) 99.

[10] Cahuzac, Ph., J. Physique 32 (1971) 499.

[11] Russel, H. N. and KING, A. S., Astrophys. J. 90 (1939) 155.

[12] Corliss, C. H. and Bozman, W. R., Experimental Transition Probabilities for Spectral Lines of Seventy Elements (N.B.S.) Monograph 53, (1962) 88.

[13] MacGillivray, J. C. and Feld, M. S., Phys. Rev. A 14 (1976) 1169. 